\title{
Perturbation facilitated two-color four-wave-mixing spectroscopy of $\mathbf{C}_{\mathbf{3}}$
}

\author{
Y. Sych, ${ }^{1}$ P. Bornhauser,${ }^{1}$ G. Knopp,${ }^{1}$ Y. Liu, ${ }^{1}$ T. Gerber,${ }^{1}$ R. Marquardt,${ }^{2}$ and P. P. Radi ${ }^{1}$ \\ ${ }^{1}$ Department General Energy Research, Paul Scherrer Institute, CH-5232 Villigen, Switzerland \\ ${ }^{2}$ Laboratoire de Chimie Quantique, Institut de Chimie, Université de Strasbourg 4, rue Blaise Pascal, \\ CS90032, 67081 Strasbourg Cedex, France
}

(Received 26 June 2013; accepted 1 October 2013; published online 21 October 2013)

\begin{abstract}
Perturbation-facilitated two-color resonant four-wave-mixing spectroscopy is realized to access the (dark) triplet manifold of the $\mathrm{C}_{3}$ molecule from the singlet $\tilde{X}^{1} \Sigma_{\mathrm{g}}^{+}$ground state. The inherent nonlinear signal dependence and coherence of the technique result in a favorable detection of the excited triplet states of interest. The observation of a newly found ${ }^{3} \Delta_{u}$ electronic state is achieved by a two-step excitation via "gate-way" levels (i.e., singlet-triplet mixed levels). Additionally, by fixing the probe laser on a transition exhibiting mainly triplet-triplet character and scanning the pump laser, we demonstrate an effective spin-filtering in a four-wave mixing measurement where only transitions to the perturber ${ }^{3} \Sigma_{\mathrm{u}}^{-}$state appear exclusively in an otherwise congested spectral range of the Comet band. Ab initio calculations of excited triplet states complement our analysis with the electronic assignment of the observed resonances. () 2013 AIP Publishing LLC. [http://dx.doi.org/10.1063/1.4825198]
\end{abstract}

\section{INTRODUCTION}

The concept of multi-photon transitions in an atom or a molecule has been predicted by Göppert-Mayer in $1931 .{ }^{1}$ Few decades later multi-photon molecular spectroscopy was widely accepted as a powerful method to observe molecular excited states. ${ }^{2,3}$ Perturbative approach multi-photon processes are treated by one or several successive transitions essentially governed by the molecular electric dipole operator $\hat{\mu}_{\alpha}$ with, respectively, $\alpha=x, y, z$. Selection rules are determined by the symmetries of the states coupled and by the symmetry of the dipole moment operator, i.e., $\Gamma_{2} \otimes \Gamma_{\hat{\mu}_{\alpha}}$ $\otimes \Gamma_{1}$. In dipole approximation two states of different spinmultiplicities cannot be coupled by the electric dipole operator except for states with spin-orbit interaction. Levels that are inaccessible by optical transitions are called dark states in contrast to the dipole allowed transitions to bright states. It is important to mention that the spectroscopically dark states can be dynamically active and play an important role in energy flow processes governing intra- and inter-molecular energy redistribution.

Perturbation effects provide ways to observe dark states on optically accessible bright states by applying double resonance techniques. ${ }^{4-6}$ In fact, perturbation facilitated opticaloptical double resonance (PFOODR) spectroscopy has been used to access dark triplet spin states from singlet ground states via singlet-triplet mixed levels as intermediate window states in two-step excitations. Mainly alkali metal dimers were studied by perturbation facilitated spectroscopy (see Refs. 7-9 and references therein). The studies have been performed by applying methods such as fluorescence excitation, ${ }^{10}$ dispersed fluorescence (DF) detection, ${ }^{9,11}$ or continuous-wave optical triple resonance spectroscopy. ${ }^{12} \mathrm{Re}-$ cently, a perturbation-assisted approach to prepare population at high vibrational states of $\mathrm{CO}\left(X^{1} \Sigma^{+}\right)$was demonstrated. ${ }^{13}$
In this work we apply, for the first time to the best of our knowledge, two-color resonant four-wave mixing (TC-RFWM) to perform PFOODR spectroscopy. We extend the earlier work on diatomic molecules by taking advantage of the coherent background-free TC-RFWM technique offering an excellent sensitivity and selectivity which is adequate to investigate the triplet manifold of the polyatomic $\mathrm{C}_{3}$ molecule exhibiting a $\tilde{X}^{1} \Sigma_{\mathrm{g}}^{+}$ground state. The high dynamic range and the favorable state selectivity of the method resulted in deperturbation studies unraveling the spin-orbit interaction of a dark state with a bright state in $\mathrm{C}_{2}{ }^{14,15}$ as well as Rydbergvalence state interactions in NO. ${ }^{16}$

The tri-carbon emission at $405 \mathrm{~nm}$ has been observed for the first time in the tail of a comet by Huggins in $1882 .{ }^{17} \mathrm{C}_{3}$ was identified in diffuse interstellar clouds through absorption in the same spectral range. ${ }^{18,19}$ In 1951 Douglas observed the $405 \mathrm{~nm}$ band at a high resolution in the laboratory. ${ }^{20} \mathrm{~A}$ spectroscopic analysis of the so-called Comet band $\tilde{A}^{1} \Pi_{\mathrm{u}}$ $-\tilde{X}^{1} \Sigma_{\mathrm{g}}^{+}$has been performed by Gausset et al. ${ }^{21}$ In addition to the significance of $\mathrm{C}_{3}$ in space, the radical is suggested as a potential precursor for soot formation in flames. ${ }^{22}$ The $\tilde{A}^{1} \Pi_{\mathrm{u}}-\tilde{X}^{1} \Sigma_{\mathrm{g}}^{+}$spectral signature appears upon irradiation of soot particles in a flame. ${ }^{23}$ Several crossed molecular beam experiments supported by ab initio calculations have analyzed possible pathways towards the formation of polyaromatic hydrocarbons (PAHs) under high-temperature flame conditions. ${ }^{24-26}$

The spectroscopy and dynamics of the linear- $\mathrm{C}_{3}\left(X^{1} \Sigma_{\mathrm{g}}^{+}\right)$ ground state were subjects of intense studies. ${ }^{22,27-31} \mathrm{~A}$ rovibrational analysis confirmed the linear ground-state structure with a low bending vibrational frequency of $63 \mathrm{~cm}^{-1} \cdot{ }^{21}$ The ground-state potential energy surface (PES) has been found to be anharmonic with a large amplitude bending motion. ${ }^{27,32}$ A number of quantum chemical calculations have been performed to examine cyclic $\mathrm{C}_{3}$ structures. ${ }^{33-35}$ The 
low-frequency bending motion is a feasible deformation path to generate the equilateral triangle. According to studies of Fueno and Taniguchi, ${ }^{34}$ the lowest ${ }^{3} \Pi$ states undergo isomerization into a cyclic structure via an asymmetric transition state. These cyclic and bent $\mathrm{C}_{3}$ structures were not observed experimentally. Though they may play an important role in the reaction pathways to the formation of PAHs, and render the $\mathrm{C}_{3}$ triplet states as an attractive object for further studies. Contrary to the singlet manifold, data available for triplet states are limited. ${ }^{36,37}$ As will be demonstrated in the following, the perturbation-facilitated two-color four-wave-mixing (PF-TC-FWM) technique is able to provide important benchmarks for the ab initio calculations of the equilibrium geometries and origins of the excited triplet states.

\section{EXPERIMENT}

The experiments are performed in a molecular beam apparatus designed for simultaneous linear and non-linear spectroscopic measurements of stable and transient molecules. ${ }^{38}$ $\mathrm{C}_{3}$ in the ground $\tilde{X}^{1} \Sigma_{\mathrm{g}}^{+}$state was prepared in the molecular beam environment by expansion of $\approx 1 \%$ acetylene diluted in Ar buffer gas through a solenoid valve (General Valve, Parker). ${ }^{39}$ Typical backing pressures are between 5 and 11 bars. The molecular pulse has a duration of about $750 \mu \mathrm{s}$. Prior to the expansion, the pulse passes through a multichannel flow-body producing a flow which is pertinent to the slit geometry of the discharge source. ${ }^{40}$ The slit-expansion geometry increases the interaction volume between the crossing area of four-wave mixing laser beams and the molecular jet (see Fig. 1). Compared to a more common pinhole expansion, the sensitivity of the method can be enhanced by nearly two orders of magnitude due to the quadratic dependence of the four-wave mixing signal on interaction length. ${ }^{41}$ A pulsed voltage of -800 to $-900 \mathrm{~V}$ and $\approx 2 \mu$ s duration is applied to the electrodes prior to the expansion zone. Trigger time delays between the discharge and the molecular beam pulse were optimized to the maximum production of $\mathrm{C}_{3}$ molecules which were probed by four-wave mixing and/or laser

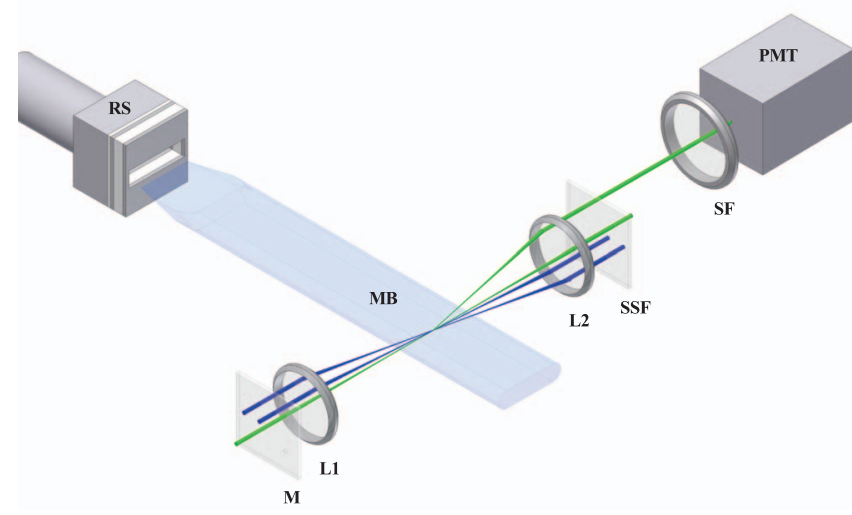

FIG. 1. Experimental arrangement: $M$ - Mask used for laser beam alignment. $L 1, L 2-1000 \mathrm{~mm}$ focal length focusing and collimating lenses, respectively. $R S$ - Radical source for $\mathrm{C}_{3} . M B$ - Molecular beam. SSF - Set of spatial filters. $S F-$ Spectral filter. $P M T$ - Photomultiplier tube with the integrated amplifier. induced fluorescence $\approx 5 \mathrm{~mm}$ downstream of the expansion region.

The optical setup for TC-RFWM is established by applying two separately pumped dye lasers (narrowscan, Radiant Dyes). The specified bandwidth of both laser systems is $\approx 0.04 \mathrm{~cm}^{-1}$. To obtain optimized spatial intensity profiles, both beams pass through spatial filters. Absolute wavelength calibration is performed for each scan step by a wavemeter (High Finesse/Ångstrom, WS6). A forward BOXCARS configuration is set up by a combination of optical components as detailed in Ref. 42 and outlined briefly in the following. A $1000 \mathrm{~mm}$ focal length achromatic objective (L1) focuses three parallel propagating laser beams to pass along the three main diagonals of a parallelepiped and cross with a small angle of $1^{\circ}$ (see Fig. 1). These beams are arranged orthogonally to the propagation direction of the molecular beam, hence, eliminating the Doppler broadening. The wave vectors, $\vec{k}_{i}$, of the interacting beams fulfill the phase-matching condition

$$
\overrightarrow{k_{1}}+\overrightarrow{k_{2}}=\overrightarrow{k_{3}}+\overrightarrow{k_{4}}
$$

and generate the signal beam $\vec{k}_{4}$ along the forth (dark) diagonal. The two laser beams of equal frequencies are usually referred to as PUMP beams $\overrightarrow{k_{1}}, \overrightarrow{k_{2}}$, and the third beam, $\vec{k}_{3}$, as PROBE beam. The signal beam is collimated by the achromatic objective $(\mathrm{L} 2)(\mathrm{FL}=1000 \mathrm{~mm})$. A set of spatial (SSF) and spectral (SF) filters remove scattered light and unwanted fluorescence along the $\approx 2 \mathrm{~m}$ propagation of the signal in freespace. Further reduction of the stray light is achieved by a $30 \mathrm{~mm}$ focal lens and a $50 \mu \mathrm{m}$ pinhole in front of a photomultiplier tube. Typically 20-30 pulses are averaged per scan step $\left(5 \times 10^{-4} \mathrm{~nm} / \mathrm{step}\right)$ of the dye-laser on the oscilloscope and transferred to the personal computer (PC) for further analysis.

\section{RESULTS AND DISCUSSION}

The intermediate levels $|i ; J p\rangle$ addressed in this work are related to the rotational $J=5$ level of parity $p$ of the $(000)$ $\tilde{A}^{1} \Pi_{\mathrm{u}}$ state which is perturbed by a nearby lying triplet state of ${ }^{3} \Sigma_{\mathrm{u}}^{-}$symmetry by spin-orbit coupling. The perturbation gives rise to mixed states exhibiting both singlet and triplet character. ${ }^{6}$ Such mixed states may be expressed as a superposition of unperturbed, zero order rovibronic states $\left|j^{(0)} ; J p\right\rangle$ with respective mixing coefficients $c_{j i}$ :

$$
|i ; J p\rangle=\sum_{j}^{n} c_{j i}\left|j^{(0)} ; J p\right\rangle,
$$

where $n$ is the number of effectively interacting states. Here, zero order states are product states characterizing an (adiabatic) electronic state of well defined spin multiplicity and a well defined vibrational state. As the total angular momentum and parity are conserved, they are equal on the right and left-hand sides of Eq. (2).

In order to identify better the intermediate levels in question here, we follow Ref. 6, but simplify the picture by including only one perturber state $\left({ }^{3} \Sigma_{\mathrm{u}}^{-}\right)$. We might then expect to 


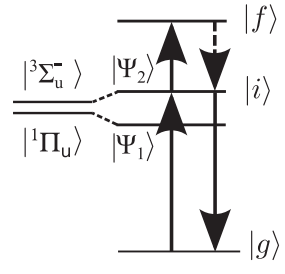

(a) UNFOLDED

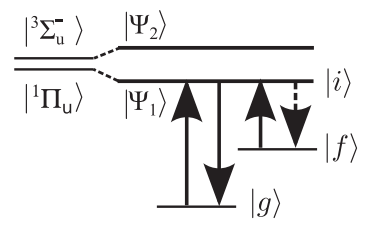

(b) SEP
FIG. 2. Energy level diagram showing the applied UNFOLDED (a) and SEP (b) double-resonance schemes by TC-RFWM. Gateway mediated intersystem-crossing from the $\tilde{X}^{1} \Sigma_{\mathrm{g}}^{+}$singlet ground state $|g\rangle$ to a triplet final state $|f\rangle$ is achieved by the UNFOLDED scheme and selecting the mixed singlet-triplet rovibronic intermediate level $|i\rangle$ with $\approx 80 \%$ triplet character $\left(\left|\psi_{2}\right\rangle\right)$. The spacing of the perturbed level pair is shown exaggerated in the figure.

have two intermediate levels:

$$
\begin{aligned}
& \left.\left|\psi_{1}\right\rangle=c_{11}\left|\tilde{A}^{1} \Pi_{\mathrm{u}}\right\rangle+\left.c_{21}\right|^{3} \Sigma_{\mathrm{u}}^{-}\right\rangle, \\
& \left.\left|\psi_{2}\right\rangle=\left.c_{22}\right|^{3} \Sigma_{\mathrm{u}}^{-}\right\rangle+c_{12}\left|\tilde{A}^{1} \Pi_{\mathrm{u}}\right\rangle,
\end{aligned}
$$

with $c_{11}=c_{22}$ and $c_{12}=-c_{21}=\sqrt{1-c_{11}^{2}}$, where we take into account the molecular constants for the perturbing ${ }^{3} \Sigma_{\mathrm{u}}^{-}$ from the deperturbation analysis of Zhang et al., ${ }^{6}$ to deduce a mixing coefficient of $\left|c_{12}\right|^{2} \approx 0.2$ for the two $J=5$ levels. For brevity, the quantum numbers for the vibrational and rotational levels, as well as the parity quantum numbers, are omitted in Eqs. (3) and (4). The corresponding interaction between the $\left|{ }^{3} \Sigma_{\mathrm{u}}^{-}\right\rangle$and $\left|\tilde{A}^{1} \Pi_{\mathrm{u}}\right\rangle$ states scales with a square of the overlap integral between vibrational wave-functions (FranckCondon factor).

The presence of the perturbation at $J=5$ represents the "gate-way" to the triplet manifold for the TC-RFWM experiments. To characterize the involved transitions, two doubleresonance schemes have been applied that are shown in Fig. 2. For the UNFOLDED ( $\Xi$-scheme), the two PUMP beams of equal frequency link the ground state $|g\rangle$ and a level $|i\rangle \in\left\{\left|\psi_{1}\right\rangle,\left|\psi_{2}\right\rangle\right\}$ of the perturbed intermediate pair. Subsequently, the PROBE beam resonant with the $|f\rangle-|i\rangle$ transition excites the higher lying final state $|f\rangle$ and generates a SIGNAL beam of the same frequency. Alternatively, the energy level scheme for the stimulated-emission pumping (SEP) type experiment ( $\Lambda$-scheme $)$ is depicted on the right-hand side. For both $\Xi$ - and $\Lambda$-schemes, the final $|f\rangle$-state is connected to the ground $|g\rangle$-state by optically allowed transitions, thus facilitating the direct assignment of the observed state by stringent two-color selection rules.

\section{A. TC-RFWM measurements via perturbed intermediate $\psi_{1}$ and $\psi_{2}$ states}

Results from TC-RFWM experiments are given in Figs. 3 and 4. The inverted spectrum in Fig. 3 is obtained by tuning the PUMP lasers of equal frequency to the perturbed P(6) transition of the Comet band (000-000) $\tilde{A}^{1} \Pi_{\mathrm{u}} \leftarrow$ $\tilde{X}^{1} \Sigma_{\mathrm{g}}^{+}$at $24669.697 \mathrm{~cm}^{-1}$ and scanning the PROBE. The excited $\left|\psi_{1}\right\rangle$ intermediate level exhibits $\approx 80 \%$ singlet character. Subsequently, the PROBE laser is scanned in the spec-

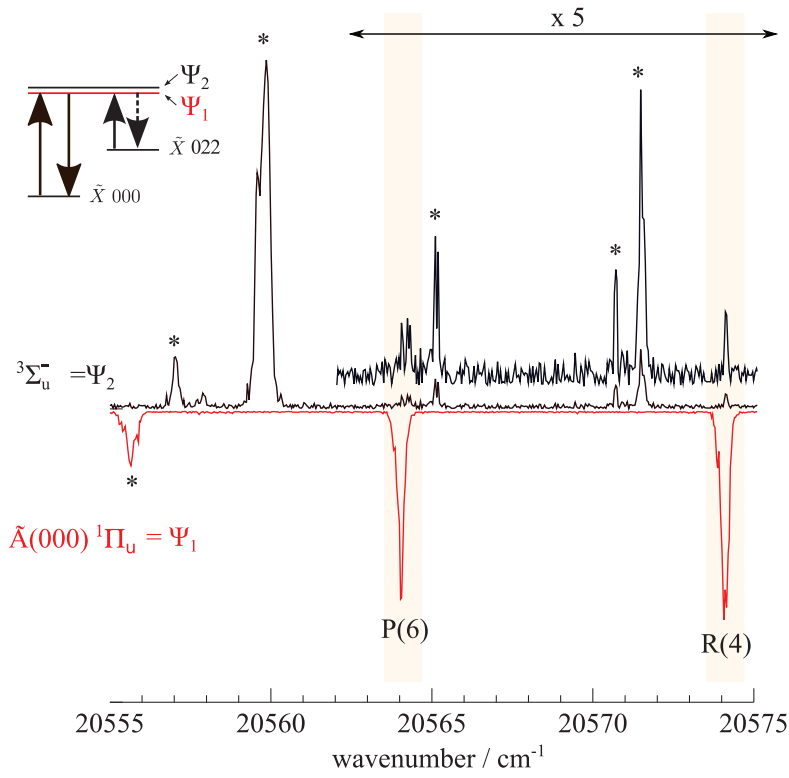

FIG. 3. SEP transitions by applying TC-RFWM from the ground state (000) $\tilde{X}^{1} \Sigma_{\mathrm{g}}^{+}$via $\left|\psi_{1}\right\rangle$ and $\left|\psi_{2}\right\rangle$ exhibiting $\approx 20 \%$ and $80 \%$ triplet character, respectively. $\mathrm{P}(6)$ and $\mathrm{R}(4)$ are SEP transitions to the vibrationally excited (022) level of the ground state $\tilde{X}^{1} \Sigma_{\mathrm{g}}^{+}$. The lower trace is shifted by $1.72 \mathrm{~cm}^{-1}$ unambiguously indicating SEP type transitions. See text for details.

tral region near $20560 \mathrm{~cm}^{-1}$. Two relatively intense SEP type transitions, i.e., $\mathrm{P}(6)$ and $\mathrm{R}(4)$ are observed. These lines connect the upper $J=5$ level of the (000) $\tilde{A}^{1} \Pi_{\mathrm{u}}$ level and the $J=4$ and $J=6$ levels of the vibrationally excited (022) $\tilde{X}^{1} \Sigma_{\mathrm{g}}^{+}$ ground state. Alternatively, excitation of the perturbing ${ }^{3} \Sigma_{\mathrm{u}}^{-}$ state $\left(\left|\psi_{2}\right\rangle\right)$, which is separated by $1.72 \mathrm{~cm}^{-1}$ only, yields a weak signal at $\mathrm{P}(6)$ and $\mathrm{R}(4)$ transitions (upper trace in Fig. 3). Extra resonances denoted by an asterisk arise because of a spectral overlap of the PUMP laser with the Q-branch of the bright $\left|\psi_{1}\right\rangle$ state.

On the other hand, the upper trace in Fig. 4 is obtained by tuning the PUMP lasers to the perturbing $\mathrm{P}(6){ }^{3} \Sigma_{\mathrm{u}}^{-}-\tilde{X}^{1} \Sigma_{\mathrm{g}}^{+}$ transition connecting the ground state with the $\left|\psi_{2}\right\rangle$ level

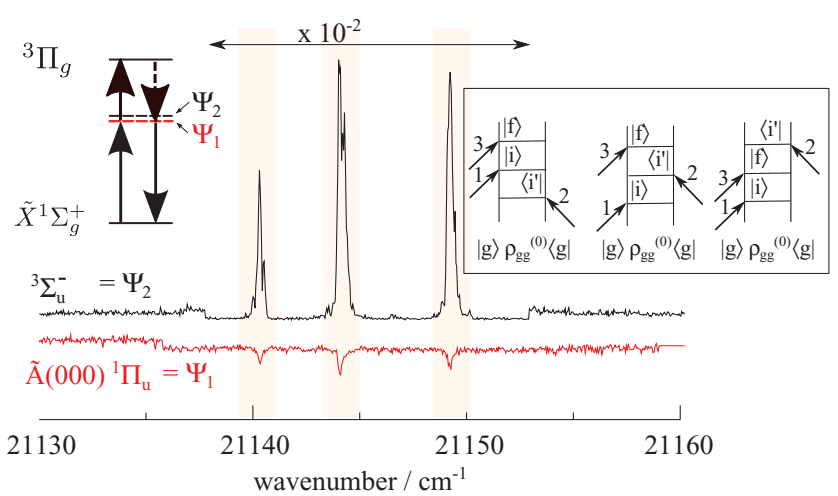

FIG. 4. UNFOLDED transitions by applying TC-RFWM from the ground state $(000) \tilde{X}^{1} \Sigma_{\mathrm{g}}^{+}$via $\left|\psi_{1}\right\rangle$ and $\left|\psi_{2}\right\rangle$ exhibiting $\approx 20 \%$ and $80 \%$ triplet character, respectively. The lower trace is shifted by $-1.72 \mathrm{~cm}^{-1}$ unambiguously indicating UNFOLDED transitions. The inset shows three relevant Feynman diagrams, assuming that only the $\mathrm{C}_{3}$ ground state is populated $\left|g>\rho_{g g}^{(0)}<g\right|$, where $\rho_{g g}^{(0)}$ is the density matrix element for the magnetic sublevel $\mathrm{m}_{g}$ at thermal equilibrium. 
exhibiting $\approx 80 \%$ triplet character. Three major transitions with substantial intensities (please note the scaling factor of $10^{-2}$ in the ordinate) are observed around $21145 \mathrm{~cm}^{-1}$. By contrast, the excitation via the $\left|\psi_{1}\right\rangle$, just $1.72 \mathrm{~cm}^{-1}$ below $\left|\psi_{2}\right\rangle$, shows that the peaks are present exhibiting, however, an intensity close to the noise level. The frequency axis for the latter is shifted by $-1.72 \mathrm{~cm}^{-1}$ indicating unambiguously an UNFOLDED transition to a high lying state. Note, that shifting the PROBE frequency axis by the $-\Delta$ or $+\Delta$, where $\Delta$ is the energy difference between the two intermediate states, provides the information on the observed double-resonance scheme, i.e., UNFOLDED or SEP, respectively.

The spectrum in Fig. 4 shows a three-line pattern measured from the $J=5$ intermediate state. The singlet-triplet mixing is sufficiently strong to perform UNFOLDED doubleresonance experiments involving intermediate states of the ${ }^{3} \Sigma_{\mathrm{u}}^{-}\left(=\left|\psi_{2}\right\rangle\right)$ for $4 \leq J \leq 16$. From these spectra, a characteristic Q-branch is recognizable by the minor energy difference relative to the neighbouring $J$-lines. The appearance of the Q-branch is a clear indication that $|f\rangle$ is a $\Pi_{\mathrm{g}}$-state. Note that Saha and Western ${ }^{31}$ performed double-resonance experiments for the Renner-Teller analysis of the excited ${ }^{1} \Delta_{\mathrm{g}}$ state and observed a similar rotational pattern for a vibronic $\Pi_{\mathrm{g}}-\Sigma_{\mathrm{u}}^{+}$ transition in the singlet manifold.

Considering the triplet character of the intermediate levels $\left|\psi_{1}\right\rangle$ and $\left|\psi_{2}\right\rangle$ of $\approx 20 \%$ and $80 \%$, respectively, and assuming a $\mathrm{P}(5), \mathrm{Q}(5)$, and $\mathrm{R}(5)$ rotational structure, the final state $|f\rangle$ is likely a triplet state of ${ }^{3} \Pi_{\mathrm{g}}$ vibronic symmetry. Further evidence of the triplet character of the $|f\rangle$ state is presented and discussed in Sec. III D below.

\section{B. The dependence of RFWM signal intensity on the intermediate state mixing coefficient $c_{J}$}

A diagrammatic perturbation theory and spherical tensor formalism were used to derive FWM signal expressions. ${ }^{43,44}$ The induced polarization of a medium is defined as $P_{R F W M}^{(3)}=N \operatorname{Tr}\left(\hat{\mu} \rho^{(3)}\right)$ a product of total number of absorbing molecules $N$, a trace operation $\operatorname{Tr}(. .$.$) over the scalar dipole$ moment operator $\hat{\mu}$, and a third-order density matrix term $\rho^{(3)}$. For a full description of $\rho^{(\mathbf{3})}$ it is important to account for the different time ordering of photon-matter interactions. ${ }^{45}$ The inset of Fig. 4 shows a set of double-sided Feynman diagrams contributing to the third order nonlinear susceptibility $\chi_{i j k l}^{(3)} \cdot 45,46$

The resonant FWM signal intensity is proportional to the modulus squared of the third-order nonlinear polarization Eq. (5):

$$
\begin{aligned}
I_{R F W M} & \propto \frac{1}{2}\left|P_{R F W M}^{(3)}\right|^{2} \\
& =\frac{1}{2}\left|\epsilon_{0} \frac{3}{2} \chi_{R F W M}^{(3)}\left(-\omega_{4}, \omega_{1}, \omega_{3},-\omega_{2}\right) E_{1} E_{2}^{*} E_{3}\right|^{2} .
\end{aligned}
$$

The spherical tensor solution of the four-photon matrix element product in $\chi_{R F W M}^{(3)}$ (for a detailed discussion, see Ref. 44 and references therein) factorizes the FWM signal expression into Einstein coefficients $B_{1}$ and $B_{3}$, geometrical factors $G_{F}^{T}\left(\boldsymbol{\epsilon}_{4}, \boldsymbol{\epsilon}_{1}, \boldsymbol{\epsilon}_{3}, \boldsymbol{\epsilon}_{2} ; J_{g}, J_{i}, J_{f}\right)$ where $\boldsymbol{\epsilon}_{1}, \boldsymbol{\epsilon}_{2}, \boldsymbol{\epsilon}_{3}$, and $\boldsymbol{\epsilon}_{4}$ are polarization unit vectors for fields $j=1,2,3$, 4, lineshape functions $L\left(\omega_{1}, \omega_{3}\right)$, laser intensities $I_{1}, I_{2}, I_{3}$, and the initial ground-state population $N_{g}$. Equation (6) allows an independent evaluation of geometrical factors and Einstein coefficients. The geometrical factors are polarization dependent and yield spatial information for magnetic sublevels $m_{J}$ of the molecular ensemble in the laboratory frame. ${ }^{47} B_{1}$ and $B_{3}$ represent the interaction of target species with the resonant laser fields in the molecular frame:

$$
\begin{aligned}
I_{F W M} \propto & N_{g}^{2} B_{1}^{2} B_{3}^{2} I_{1} I_{2} I_{3} \\
& \times L\left(\omega_{1}, \omega_{3}\right)^{2} G_{F}^{T}\left(\boldsymbol{\epsilon}_{4}, \boldsymbol{\epsilon}_{1}, \boldsymbol{\epsilon}_{3}, \boldsymbol{\epsilon}_{2} ; J_{g}, J_{i}, J_{f}\right)^{2} .
\end{aligned}
$$

Einstein coefficients are written via a square of the reduced matrix element $\left\langle\left\|\mu^{(1)}\right\|\right\rangle$ for the involved transitions $|g\rangle$, $|i\rangle \in\left\{\left|\psi_{1}\right\rangle,\left|\psi_{2}\right\rangle\right\},|f\rangle$ :

$$
\begin{aligned}
& B_{1}=B_{g, i}=\frac{2 \pi^{2}}{3 \epsilon_{0} h^{2}} \frac{\left|\left\langle i\left\|\mu^{(1)}\right\| g\right\rangle\right|^{2}}{2 J_{g}+1}, \\
& B_{3}=B_{i, f}=\frac{2 \pi^{2}}{3 \epsilon_{0} h^{2}} \frac{\left|\left\langle f\left\|\mu^{(1)}\right\| i\right\rangle\right|^{2}}{2 J_{i}+1} .
\end{aligned}
$$

The relevant diagrams contributing to $\chi_{R F W M}^{(3)}$ are shown in the inset of Fig. 4. The first two diagrams (from the left to the right) describe the PROBE beam scattering on the PUMPinduced population grating in the intermediate state $\left|\psi_{1,2}\right\rangle$. The third diagram may be interpreted as a coherence between $|g\rangle$ and $|f\rangle$ induced by a sequential absorption of a PUMP and a PROBE photon. In this case, the RFWM signal is emitted when the second PUMP photon is absorbed to the intermediate $\left|\psi_{1,2}\right\rangle$ state. For all three diagrams, the signal emerges in the "phase-matched" direction, Eq. (1), and has the frequency $\omega_{4}=\omega_{1}+\omega_{3}-\omega_{2}\left(\omega_{1}=\omega_{2} \neq \omega_{3}\right)$.

The optimal conditions to observe $|f\rangle$ levels of triplet spin-symmetry through the gateway $\left|\psi_{1}\right\rangle$ or $\left|\psi_{2}\right\rangle$ states are determined by inserting Eqs. (7) and (8) and Eqs. (3) and (4) into Eq. (6), this gives the eighth power of the mixing coefficients in the signal intensity. Considering the PUMP resonantly tuned to a $\left|\psi_{2}\right\rangle$ state with $\approx 80 \%$ triplet character and a PROBE resonant to a triplet $|f\rangle$ final state, the FWM signal is scaled by a factor of $\left(\left|c_{12}\right|^{8}=0.2^{4}\right) \times\left(\left|c_{22}\right|^{8}=0.8^{4}\right)$, respectively. Similar reasoning holds for intermediate excitation via $\psi_{1}$ and transitions within the singlet manifold.

It is important that the RFWM signal depends on the eighth power of the mixing coefficient. Linear methods such as absorption spectroscopy have a quadratic dependence on the mixing coefficient. Stimulated emission pumping and other double-resonance methods based on sequential absorption of two photons have quadratic dependence on $c_{J}$ for each consecutive step. The four-wave mixing eighth-power functional relationship between $c_{J}$ and the observable signal facilitates a spin-symmetry assignment of a final state (e.g., by the observation of line-strength propensities for corresponding intermediate states). The eighth-power law appears to be of an exceptional advantage for the selectivity of the method which makes it rather insensitive to small perturbations. Although, in order to measure $J$-dependent spectra containing spin-forbidden transitions the potential energy surfaces 


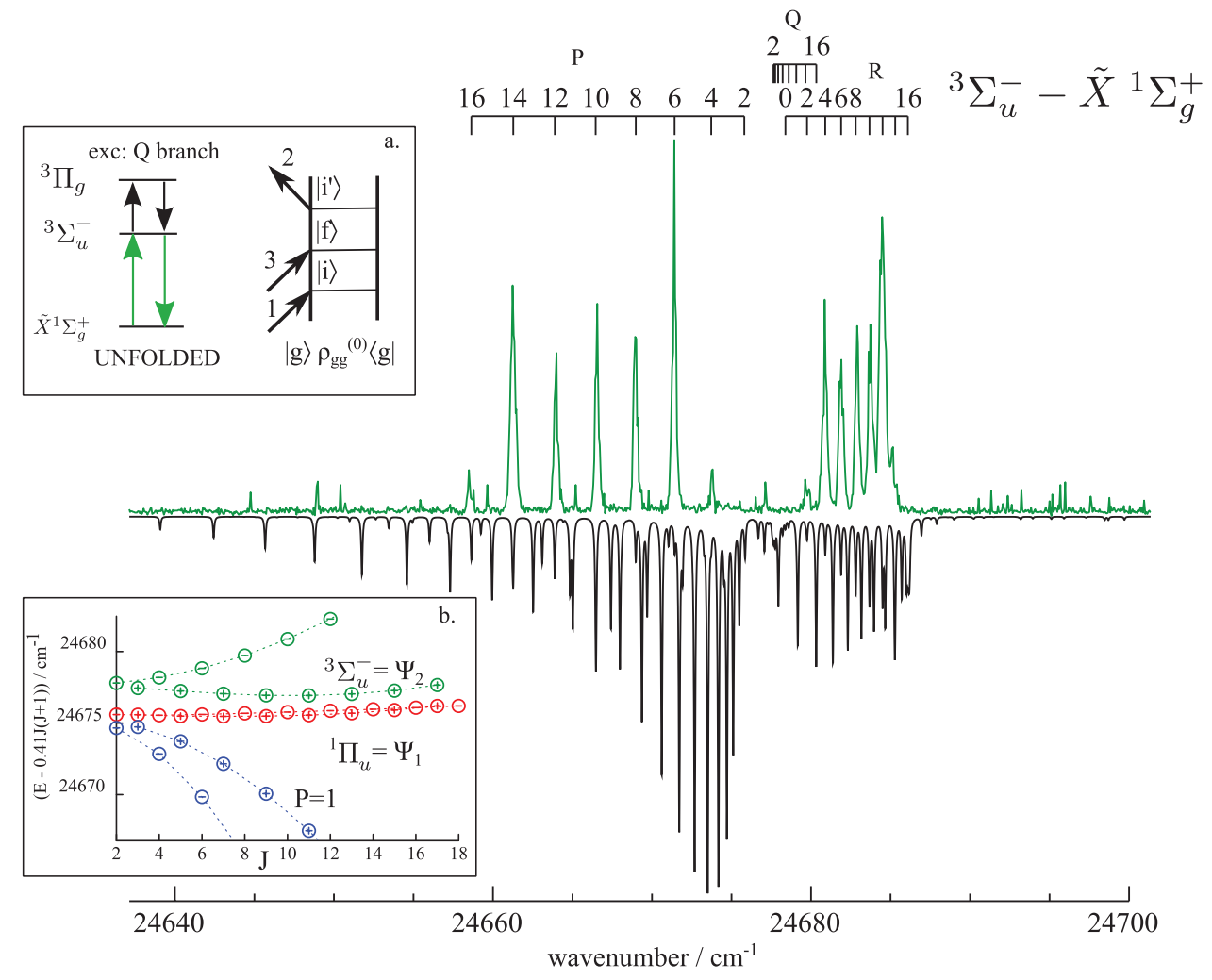

FIG. 5. PFOODR spectrum by tuning the PROBE laser to several overlapping Q lines in the ${ }^{3} \Pi_{\mathrm{g}}{ }^{3} \Sigma_{\mathrm{u}}^{-}\left(=\left|\psi_{2}\right\rangle\right)$ vibronic system and scanning the PUMP lasers. Exclusively transitions from the ground state $\tilde{X}^{1} \Sigma_{\mathrm{g}}^{+}$to the perturbing ${ }^{3} \Sigma_{\mathrm{u}}^{-}$state are observed showing further evidence that the final state exhibits substantial triplet character. For comparison, a simulation of the Comet band absorption spectrum is shown at the bottom where the significant features are governed by the $\tilde{A}^{1} \Pi_{\mathrm{u}} \leftarrow \tilde{X}^{1} \Sigma_{\mathrm{g}}^{+}$transition. The inset (a) shows the level scheme and the coherence diagram contributing to a RFWM signal. The inset (b) shows potential energy surfaces of interacting ${ }^{3} \Sigma_{\mathrm{u}}^{-}$and $\tilde{A}^{1} \Pi_{\mathrm{u}}$ electronic states $v$ s. $J$. Symmetry (even/odd) $-\oplus$ : e $(+/-)$ and $\ominus$ : $\mathrm{f}(-/+)$. Note that the unknown electronic $P=1$ state perturbs $\tilde{A}^{1} \Pi_{\mathrm{u}}$ only in the $1 \leq J \leq 3$ range. $^{6}$

of two intermediate states should strongly interact in a wide range of rotational levels (e.g., in the present work spin-orbit interaction is sufficiently strong in the $4 \leq J \leq 16$ range, see Fig. 5(b)). The challenge of weak spin-orbit interaction might be circumvented by the selective Stark/Zeeman tuning of the magnetic sublevel $m_{J}$ of a dark state to interact with the $m_{J}$ of a bright state.

\section{Saturation behavior of the intermediate states $\psi_{1}$ and $\psi_{2}$}

For the applied pulse energies one should be cautious of the signal saturation behavior which consequently affects the intensity ratio between chosen $\left|\psi_{1}\right\rangle$ and $\left|\psi_{2}\right\rangle$ intermediate states. Considering a strong PUMP beam coupling the ground state with a dark $\left|\psi_{2}\right\rangle$ or a bright $\left|\psi_{1}\right\rangle$ state with the transition dipole moments evaluated in Table I, the contribution of the PUMP beam to the four-wave mixing signal intensity is balanced by saturation for both $\psi_{j}-\tilde{X}^{1} \Sigma_{\mathrm{g}}^{+}$transitions $(j=1,2)$. The estimated spectral irradiance which saturates the PUMP transition is at least three orders of magnitude inferior to the spectral irradiance applied in current experiments. This significant saturation level is also reflected in the observed power-broadened linewidth of $\approx 12 \mathrm{GHz}$. A weak PROBE explores the intermediate state population grating giving rise to a $\left|c_{22}\right|^{8}$ and a $\left|c_{21}\right|^{8}$ signal intensity depen- dence of the ${ }^{3} \Pi_{\mathrm{g}}-\psi_{2}$ and ${ }^{3} \Pi_{\mathrm{g}}-\psi_{1}$ transitions, respectively. The RFWM signal intensity ratios for the observed P, Q, and $\mathrm{R}$ branch resonances to the ${ }^{3} \Pi_{\mathrm{g}}$ state (Fig. 4) are on the order of 250 for two intermediate states $\left|\psi_{1}\right\rangle$ and $\left|\psi_{2}\right\rangle$. This value is in a qualitative agreement with the ratio of the singlet-triplet mixing coefficients $\left|c_{22}\right|^{8} /\left|c_{21}\right|^{8}=2^{8}=256$.

TABLE I. Oscillator strength, transition dipole moment, and saturation intensities.

\begin{tabular}{lcccc}
\hline \hline Line & $\begin{array}{c}\text { Life-time } \\
\mathrm{ns}\end{array}$ & $\begin{array}{c}\text { Oscillator } \\
\text { strength } \\
f_{g i} \mathrm{~b}\end{array}$ & $\begin{array}{c}\text { Dipole } \\
\text { moment } \\
\times 10^{-29}(\mathrm{C} \mathrm{m})\end{array}$ & $\begin{array}{c}\text { Saturation } \\
\text { intensity } \\
\left(\mathrm{W} / \mathrm{m}^{2}\right)^{\mathrm{c}}\end{array}$ \\
\hline$P(4) \tilde{A}^{1} \Pi_{\mathrm{u}}$ & 202 & 0.0243 & 0.3416 & 62.01 \\
$P(4)^{3} \Sigma_{\mathrm{u}}^{-}$ & 786 & 0.0063 & 0.1732 & 15.93 \\
$R(2) \tilde{A}^{1} \Pi_{\mathrm{u}}$ & 223 & 0.0221 & 0.3251 & 56.17 \\
$R(2)^{3} \Sigma_{\mathrm{u}}^{-}$ & 2190 & 0.0022 & 0.1037 & 5.72 \\
\hline \hline
\end{tabular}

${ }^{\text {a } B a s e d ~ o n ~ t h e ~ l i f e-t i m e ~ m e a s u r e m e n t s ~ o f ~ t h e ~} \tilde{A}^{1} \Pi_{\mathrm{u}}-\tilde{X}^{1} \Sigma_{\mathrm{g}}^{+}$band with an inclusion of the perturber ${ }^{3} \Sigma_{\mathrm{u}}^{-}$state, Ref. 6.

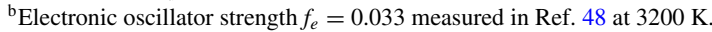

${ }^{\mathrm{c}}$ If saturation intensity is calculated for the Rabi frequency $\Omega_{s a t}=\sqrt{\Delta \nu_{L} \Gamma_{0}}$, when a laser bandwidth is large compared to a coherence life-time $\Delta v_{L} \gg \Gamma_{i g}$. Saturation intensity for the PUMP transition is $\approx 7.5 \mathrm{~kW} / \mathrm{m}^{2}$. This value is about three orders of magnitude lower than experimentally applied PUMP intensities. See text and Ref. 49 for details. 


\section{D. “The spin filter” TC-RFWM measurement}

Further evidence for the triplet character of the final state $|f\rangle$, which is observed by applying UNFOLDED PF-RFWM via the $\left|\psi_{2}\right\rangle$ intermediate state, is shown in Fig. 5. Here, PF-RFWM spectroscopy is performed by tuning the PROBE laser to the narrowly spaced Q-branch region and scanning the PUMP lasers in the spectral region of the Comet band $(000-$ 000) $\tilde{A}^{1} \Pi_{\mathrm{u}} \leftarrow \tilde{X}^{1} \Sigma_{\mathrm{g}}^{+}$. The RFWM coherence is described by the double-sided diagram shown in Fig. 5(a). In the spectrum, exclusively $\mathrm{P}$ and $\mathrm{R}$ transitions are observed with significant intensities that involve the intermediate perturbing state ${ }^{3} \Sigma_{\mathrm{u}}^{-}$ with $J=4-16$. For comparison, a typical absorption spectrum of the Comet band is simulated and shown inverted in the lower trace. The observation is rationalized in a straightforward manner by assuming an intermediate level labeling of several overlapping Q-lines in the ${ }^{3} \Pi_{\mathrm{g}}{ }^{3} \Sigma_{\mathrm{u}}^{-}\left(|f\rangle-\left|\psi_{2}\right\rangle\right)$ system which are addressed simultaneously by the PROBE laser. It is important to mention that the perturber transitions are generally weak and difficult to observe in common absorption spectroscopy. In fact, a detailed analysis has been achieved only recently by applying cavity ring-down and time-resolved LIF measurements. ${ }^{6,50}$

It should be noted that the intensity distribution of observed rotational lines does not reflect the population distribution among rotational levels of the $\mathrm{C}_{3}$ ground state. There are two reasons for a non-thermal $J$-dependence of the measured intensities in the spectrum of Fig. 5. On the one hand, the rovibronic spectrum is truncated by the eighth power of the mixing coefficient $c_{J}$ (vide supra) which in turn depends on the interaction of $\tilde{A}^{1} \Pi_{\mathrm{u}}$ and ${ }^{3} \Sigma_{\mathrm{u}}^{-}$(Fig. 5(b)). On the other hand, the partial spectral overlap of the PROBE laser with the narrowly spaced Q-branch limits the number of $J$ levels that are observed.

\section{E. $A b$ initio calculations of the $\mathbf{C}_{3}$ triplet manifold}

To shed more light on the $\mathrm{C}_{3}$ triplet manifold, $a b$ initio calculations have been performed at the multi-reference configuration interaction (MRCI) level with the MOLPRO package. ${ }^{51}$ Active orbital spaces and reference states were obtained by applying complete active space self-consistent field (CASSCF) theory. ${ }^{52}$ The MRCI calculations were conducted with equal bond lengths of $r=1.2950 \AA{ }^{31}$ The internally contracted MRCI method of Werner and Knowles ${ }^{53-56}$ was used in a complete active space (CAS) of 12 electrons in 12 orbitals. This CAS is the complete valence active space. The results of the cluster Davidson corrected transition energies $\Delta E$ obtained with the aug-cc-pVTZ basis set are summarized in Table II. We estimate the accuracy of the calculated values to be better than $200 h c \mathrm{~cm}^{-1}$ (where $h$ is the Planck constant and $c$ is the speed of light in vacuum), both with respect to the basis size used as well as with regard to the inclusion of additional electronic correlation.

The newly measured excited state of ${ }^{3} \Pi_{\mathrm{g}}$ vibronic symmetry (i.e., $\Gamma_{\text {vib }} \otimes \Gamma_{\text {el }}$ ) can be assigned to a few possible final electronic states: $\Delta_{\mathrm{u}},{ }^{3} \Sigma_{\mathrm{u}}^{-}$, or ${ }^{3} \Pi_{\mathrm{g}}$ appearing in the vicinity of $45000 \mathrm{~cm}^{-1}$ (Table II). Selection rules prohibit transitions between $\mathrm{u} \leftrightarrow \mathrm{u}$ (ungerade) states. As a consequence,
TABLE II. Electronic term values of $\mathrm{C}_{3}$ calculated at the MRCI level of theory, at a fixed bond length of $r=1.2950 \AA$ and linear geometry.

\begin{tabular}{|c|c|c|c|}
\hline $\begin{array}{l}\text { Electronic } \\
\text { state }\end{array}$ & $\begin{array}{l}\text { Dominant electronic } \\
\text { configuration }\end{array}$ & $\begin{array}{c}\text { Calc. } \\
\Delta E^{\mathrm{a}}\left(h c \mathrm{~cm}^{-1}\right)\end{array}$ & $\begin{array}{c}\text { Obs. } \\
T_{o}^{\mathrm{b}}\left(\mathrm{cm}^{-1}\right)\end{array}$ \\
\hline$\tilde{X}^{1} \Sigma_{\mathrm{g}}^{+}$ & $\ldots 1 \pi_{\mathrm{u}}^{4} 4 \sigma_{\mathrm{g}}^{2} 3 \sigma_{\mathrm{u}}^{2}$ & 0 & $\ldots$ \\
\hline$\tilde{a}^{3} \Pi_{\mathrm{u}}$ & $\ldots 1 \pi_{\mathrm{u}}^{4} 4 \sigma_{\mathrm{g}}^{2} 3 \sigma_{\mathrm{u}}^{1} 1 \pi_{\mathrm{g}}^{1}$ & 17205 & $16940^{c}$ \\
\hline$\tilde{b}^{3} \Pi_{\mathrm{g}}$ & $\ldots 1 \pi_{\mathrm{u}}^{4} 4 \sigma_{\mathrm{g}}^{1} 3 \sigma_{\mathrm{u}}^{2} 1 \pi_{\mathrm{g}}^{1}$ & 23696 & $23390^{\mathrm{d}}$ \\
\hline${ }^{1} \Pi_{\mathrm{u}}$ & $\ldots 1 \pi_{\mathrm{u}}^{4} 4 \sigma_{\mathrm{g}}^{2} 3 \sigma_{\mathrm{u}}^{1} 1 \pi_{\mathrm{g}}^{1}$ & 24997 & $24675^{\mathrm{e}}$ \\
\hline${ }^{3} \Sigma_{\mathrm{u}}^{+}$ & $\ldots 1 \pi_{\mathrm{u}}^{3} 4 \sigma_{\mathrm{g}}^{2} 3 \sigma_{\mathrm{u}}^{2} 1 \pi_{\mathrm{g}}^{1}$ & 26120 & $\cdots$ \\
\hline${ }^{3} \Delta_{\mathrm{u}}$ & $\ldots 1 \pi_{\mathrm{u}}^{3} 4 \sigma_{\mathrm{g}}^{2} 3 \sigma_{\mathrm{u}}^{2} 1 \pi_{\mathrm{g}}^{1}$ & 29452 & $\cdots$ \\
\hline${ }^{3} \Sigma_{\mathrm{u}}^{-}$ & $\ldots 1 \pi_{\mathrm{u}}^{3} 4 \sigma_{\mathrm{g}}^{2} 3 \sigma_{\mathrm{u}}^{2} 1 \pi_{\mathrm{g}}^{1}$ & 31164 & $\ldots$ \\
\hline${ }^{1} \Delta_{\mathrm{u}}$ & $\ldots 1 \pi_{\mathrm{u}}^{3} 4 \sigma_{\mathrm{g}}^{2} 3 \sigma_{\mathrm{u}}^{2} 1 \pi_{\mathrm{g}}^{1}$ & $32123^{\mathrm{f}}$ & $\cdots$ \\
\hline${ }^{1} \Sigma_{\mathrm{u}}^{-}$ & $\ldots 1 \pi_{\mathrm{u}}^{3} 4 \sigma_{\mathrm{g}}^{2} 3 \sigma_{\mathrm{u}}^{2} 1 \pi_{\mathrm{g}}^{1}$ & $32325^{\mathrm{f}}$ & $\ldots$ \\
\hline${ }^{1} \Pi_{\mathrm{g}}$ & $\begin{array}{l}\ldots 1 \pi_{\mathrm{u}}^{4} 4 \sigma_{\mathrm{g}}^{1} 3 \sigma_{\mathrm{u}}^{2} 1 \pi_{\mathrm{g}}^{1} \\
+. .1 \pi_{\mathrm{u}}^{4} 4 \sigma_{\mathrm{g}}^{2} 3 \sigma_{\mathrm{u}}^{1} 1 \pi_{\mathrm{g}}^{1}\end{array}$ & 32411 & $\cdots$ \\
\hline${ }^{3} \Sigma_{\mathrm{g}}^{-}$ & $\begin{array}{l}\ldots 1 \pi_{\mathrm{u}}^{4} 4 \sigma_{\mathrm{g}}^{2} 3 \sigma_{\mathrm{u}}^{0} 1 \pi_{\mathrm{g}}^{2} \\
+. .1 \pi_{\mathrm{u}}^{4} 4 \sigma_{\mathrm{g}}^{0} 3 \sigma_{\mathrm{u}}^{2} 1 \pi_{\mathrm{g}}^{2}\end{array}$ & 34695 & $\ldots$ \\
\hline${ }^{1} \Delta_{\mathrm{g}}$ & $\begin{array}{l}\ldots 1 \pi_{\mathrm{u}}^{4} 4 \sigma_{\mathrm{g}}^{2} 3 \sigma_{\mathrm{u}}^{0} 1 \pi_{\mathrm{g}}^{2} \\
+. .1 \pi_{\mathrm{u}}^{4} 4 \sigma_{\mathrm{g}}^{0} 3 \sigma_{\mathrm{u}}^{2} 1 \pi_{\mathrm{g}}^{2}\end{array}$ & 42310 & $42059^{g}$ \\
\hline${ }^{3} \Delta_{\mathrm{u}}$ & $\ldots 1 \pi_{\mathrm{u}}^{4} 4 \sigma_{\mathrm{g}}^{1} 3 \sigma_{\mathrm{u}}^{1} 1 \pi_{\mathrm{g}}^{2}$ & 44859 & $45846^{\mathrm{h}}$ \\
\hline${ }^{3} \Sigma_{\mathrm{u}}^{-}$ & $\ldots 1 \pi_{\mathrm{u}}^{4} 4 \sigma_{\mathrm{g}}^{1} 3 \sigma_{\mathrm{u}}^{1} 1 \pi_{\mathrm{g}}^{2}$ & 46070 & $\cdots$ \\
\hline${ }^{3} \Pi_{g}$ & $\ldots 1 \pi_{\mathrm{u}}^{3} 4 \sigma_{\mathrm{g}}^{2} 3 \sigma_{\mathrm{u}}^{1} 1 \pi_{\mathrm{g}}^{2}$ & 47335 & $\ldots$ \\
\hline
\end{tabular}

${ }^{\mathrm{a}} \Delta E=E-E\left(\tilde{X}^{1} \Sigma_{\mathrm{g}}^{+}\right)$at the given geometry, where $E\left(\tilde{X}^{1} \Sigma_{\mathrm{g}}^{+}\right)=-113.8420 E_{\mathrm{h}}\left(E_{\mathrm{h}}\right.$ $\approx 4.359710^{-18} \mathrm{~J}$ is the hartree); for the calculated values a $(12,12)$ CAS and the augcc-pVTZ basis were used as implemented in Ref. 51, and they include the rotated frame Davidson correction.

${ }^{\mathrm{b}}$ Vertical excitation energy.

${ }^{\mathrm{c}}$ Reference 57.

${ }^{\mathrm{d}}$ Reference 36 .

e Reference 21 .

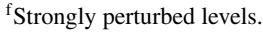

${ }^{\mathrm{g}}$ Reference 31 .

${ }^{\mathrm{h}}$ This work. The observed state is likely a $\left(0 n^{1} 0\right)$ vibrational level of the ${ }^{3} \Delta_{\mathrm{u}}$ resulting in

${ }^{3} \Pi_{\mathrm{g}}$ vibronic symmetry (see text), where $n$ is the odd number of bent vibrational quanta.

no direct transitions to the (000) vibrational level of either the ${ }^{3} \Delta_{\mathrm{u}}$ or the ${ }^{3} \Sigma_{\mathrm{u}}^{-}$states are possible. However, transitions might be allowed by considering the direct product with the vibrational state symmetry. When odd numbers of bent vibrations are excited $(0 n 0), n=1,3, .$. both $\Delta_{\mathrm{u}}$ and $\Sigma_{\mathrm{u}}^{-}$electronic states are dipole coupled to the intermediate state through the $\Gamma_{\Pi_{\mathrm{u}}} \otimes \Gamma_{\Delta_{\mathrm{u}}}=\Gamma_{\Pi_{\mathrm{g}}} \oplus \Gamma_{\Phi_{\mathrm{g}}}$ and $\Gamma_{\Pi_{\mathrm{u}}} \otimes \Gamma_{\Sigma_{\mathrm{u}}^{-}}=\Gamma_{\Pi_{\mathrm{g}}}$ symmetry product. Note that no a priori knowledge on the electronic symmetry of the intermediate ${ }^{3} \Sigma_{\mathrm{u}}^{-}$state is known. Assuming the $\Gamma_{(050)} \otimes \Gamma_{\mathrm{el}}={ }^{3} \Sigma_{\mathrm{u}}^{-}$perturber state as suggested by Zhang et al., ${ }^{6}$ the transition from $\tilde{b}^{3} \Pi_{\mathrm{g}}$ to either $\Delta_{\mathrm{u}}$ or ${ }^{3} \Sigma_{\mathrm{u}}^{-}$is electronically allowed.

We should also mention that from a preliminary analysis of the potential energy surfaces of the excited states, we found the occurrence of several crossings in the vicinity of the ${ }^{3} \Delta_{\mathrm{u}}$ electronic state. It is possible that the Renner-Teller effect is quite important in this region. A more careful analysis of the rovibronic spectrum including a correct treatment of the Renner-Teller hamiltonian is clearly in place in order to have a more conclusive interpretation of the detailed structure of the final state. While such an analysis is foreseen in the forthcoming paper, we may conclude that the rough 
TABLE III. Effective molecular constants for the ${ }^{3} \Pi_{\mathrm{g}}$ vibronic level. All values are in $\mathrm{cm}^{-1}$. The origin, $T$, is relative to the $(0,0,0) \tilde{X}^{1} \Sigma_{\mathrm{g}}^{+}$state. For comparison, constants for the two known triplet states $\tilde{a}$ and $\tilde{b}$ are reproduced from Ref. 36.

\begin{tabular}{lccc}
\hline \hline Parameter & ${ }^{3} \Pi_{\mathrm{g}}$ (vibronic) & $\tilde{b}^{3} \Pi_{\mathrm{g}}$ & $\tilde{a}^{3} \Pi_{\mathrm{u}}$ \\
\hline$T$, origin & $45846.348(3711)$ & & \\
$A$ & $12.817(1860)$ & $13.919(44)$ & $13.556(43)$ \\
$B$ & $0.41858(176)$ & $0.424524(22)$ & $0.416957(19)$ \\
$o$ & $-0.1253(94)$ & $0.581(54)$ & $0.595(54)$ \\
$p \times 10^{3}$ & $12.4(32)$ & $-2.2(12)$ & $-2.2(12)$ \\
\hline \hline
\end{tabular}

assignments from the present $a b$ initio calculations and rotational analysis to be discussed in Sec. III F are correct.

\section{F. Rotational analysis of the observed ${ }^{3} \Pi_{g}$ state}

The singlet-triplet mixing is sufficiently strong to perform UNFOLDED double-resonance experiments involving intermediate states of the ${ }^{3} \Sigma_{\mathrm{u}}^{-}\left(=\left|\psi_{2}\right\rangle\right)$ for $4 \leq J \leq 16$. For each accessed intermediate level $J$ a spectrum according to Fig. 4 is obtained displaying a P, Q, and R line in accordance with the selection rules for a $\Pi \leftarrow \Sigma$ transition.

A preliminary $\mathrm{fit}^{58}$ of the unambiguously assigned rotational structure by measuring 32 transitions in the ${ }^{3} \Pi_{\mathrm{g}}-{ }^{3} \Sigma_{\mathrm{u}}^{-}\left(=\left|\psi_{2}\right\rangle\right)$ system yields the effective molecular constants in Table III with an average error of $0.029 \mathrm{~cm}^{-1}$. The rotational constant $B$ suggests a bond-length similar to that of the $\tilde{a}^{3} \Pi_{\mathrm{u}}$ state. From preliminary calculations of the potential energy surfaces, we estimate that the equilibrium bond distance in the electronic ${ }^{3} \Pi_{\mathrm{g}}$ state should be at significantly larger values than that of the $\tilde{a}^{3} \Pi_{\mathrm{u}}$ state. The rotational constant in the ${ }^{3} \Pi_{\mathrm{g}}$ state therefore would not agree with the experimentally observed value and we might rule out this state as a possible candidate for being the final $|f\rangle$ state. The spin-orbit constant $A$ determined from the rotational spectra is non-zero. This rules out the ${ }^{3} \Sigma_{\mathrm{u}}^{-}$state. However, note that an accurate evaluation of $A$ requires measurements of several spin sub-states. Nevertheless, from the non-zero spin-orbit coupling constant we conclude that the observed resonances rather belong to the ${ }^{3} \Delta_{\mathrm{u}}$ electronic state. The $\Lambda$-type doubling parameters of ${ }^{3} \Delta_{\mathrm{u}}$ state are quite different compared to the known ${ }^{3} \Pi$ states (Table III).

\section{CONCLUSIONS}

Perturbation-facilitated-RFWM spectroscopy is a versatile technique capable of measuring topographic maps of excited triplet states of the $\mathrm{C}_{3}$ molecule exhibiting a singlet ground state. By utilizing the nonlinear signal dependence on the mixing coefficient $c_{J}$ of the intermediate state ("gate way"), it is possible to characterize transitions to final electronic states of a different spin multiplicity with respect to the initial state. The reported technique can be applied to any molecular system with states mixed by spin-orbit interaction.

The importance of the excited triplet states for the isomerization of the linear tri-carbon to an equilateral triangle structure has been pointed out by Fueno and Taniguchi ${ }^{34}$ and others. ${ }^{33,35}$ The lowest equilateral triplet state is expected at $1.0-1.88 \mathrm{eV}$ above the linear ground state, thus it would form the lowest excited state of $\mathrm{C}_{3}$. This state could be formed by important radical reactions, like $\mathrm{C}+\mathrm{C}_{2} \mathrm{H}$ and $\mathrm{CH}+\mathrm{C}_{2} \cdot{ }^{59}$

High-level $a b$ initio calculations of the triplet PES as well as the perturbation facilitated dispersed LIF experiments from the newly found ${ }^{3} \Pi_{\mathrm{g}}$ vibronic level, which is tentatively assigned to the ${ }^{3} \Delta_{\mathrm{u}}$ electronic state, are under way in our laboratories. These studies shed more light on the low lying triplet manifold and will be a subject of a future report.

\section{ACKNOWLEDGMENTS}

We thank B. Murali Krishna. This work is supported by the Swiss National Science Foundation (Grant Nos. 200020_124542 and 200020_146387), and from CNRS, Région Alsace and the French Ministry for Research.

${ }^{1}$ M. Göppert-Mayer, Ann. Phys. 401, 273 (1931).

${ }^{2}$ W. L. Peticolas, Annu. Rev. Phys. Chem. 18, 233 (1967).

${ }^{3}$ W. M. McClain, Acc. Chem. Res. 7, 129 (1974).

${ }^{4}$ H. Lefebvre-Brion and R. W. Field, in The Spectra and Dynamics of Diatomic Molecules (Academic Press, San Diego, 2004), pp. 87-231.

${ }^{5}$ L. L. Lohr, Jr., Coord. Chem. Rev. 8, 241 (1972).

${ }^{6}$ G. Zhang, K.-S. Chen, A. J. Merer, Y.-C. Hsu, W.-J. Chen, S. Shaji, and Y.-A. Liao, J. Chem. Phys. 122, 244308 (2005).

${ }^{7}$ L. Li and R. W. Field, J. Phys. Chem. 87, 3020 (1983).

${ }^{8}$ X. Xie and R. Field, J. Mol. Spectrosc. 117, 228 (1986).

${ }^{9}$ H. L. Dai, Molecular Dynamics and Spectroscopy by Stimulated Emission Pumping (World Scientific, 1995).

${ }^{10}$ F. Xie, D. Li, L. Li, R. W. Field, and S. Magnier, Chem. Phys. Lett. 431, 267 (2006).

${ }^{11}$ J. Magnes, E. Ahmed, C. Goldberg, A. Lyyra, S. Magnier, M. AubertFrécon, Y. Liu, and L. Li, J. Mol. Spectrosc. 221, 72 (2003).

${ }^{12}$ G. Jong, L. Li, T.-J. Whang, W. Stwalley, J. A. Coxon, M. Li, and A. Lyyra, J. Mol. Spectrosc. 155, 115 (1992).

${ }^{13}$ N. Bartels, T. Schäfer, J. Hühnert, R. W. Field, and A. M. Wodtke, J. Chem. Phys. 136, 214201 (2012).

${ }^{14}$ P. Bornhauser, G. Knopp, T. Gerber, and P. Radi, J. Mol. Spectrosc. 262, 69 (2010).

${ }^{15}$ P. Bornhauser, Y. Sych, G. Knopp, T. Gerber, and P. P. Radi, J. Chem. Phys. 134, 044302 (2011).

${ }^{16}$ E. F. McCormack, P. M. Dehmer, J. L. Dehmer, and S. T. Pratt, J. Chem. Phys. 102, 4740 (1995).

${ }^{17}$ W. Huggins, Proc. R. Soc. London 33, 1 (1882).

${ }^{18}$ J. P. Maier, N. M. Lakin, G. A. H. Walker, and D. A. Bohlender, Astrophys. J. 553, 267 (2001).

${ }^{19}$ T. Oka, J. A. Thorburn, B. J. McCall, S. D. Friedman, L. M. Hobbs, P. Sonnentrucker, D. E. Welty, and D. G. York, Astrophys. J. 582, 823 (2003).

${ }^{20}$ A. E. Douglas, Astrophys. J. 114, 466 (1951).

${ }^{21}$ L. Gausset, G. Herzberg, A. Lagerqvist, and B. Rosen, Astrophys. J. 142, 45 (1965).

${ }^{22}$ E. A. Rohlfing, J. Chem. Phys. 91, 4531 (1989).

${ }^{23}$ F. Goulay, L. Nemes, P. E. Schrader, and H. A. Michelsen, Mol. Phys. 108, 1013 (2010).

${ }^{24}$ R. I. Kaiser, T. N. Le, T. L. Nguyen, A. M. Mebel, N. Balucani, Y. T. Lee, F. Stahl, P. v. R. Schleyer, and H. F. Schaefer III, Faraday Discuss. 119, 51 (2001).

${ }^{25}$ X. Gu, Y. Guo, A. M. Mebel, and R. I. Kaiser, Chem. Phys. Lett. 449, 44 (2007).

${ }^{26}$ J. Szczepanski, H. Wang, and M. Vala, Chem. Phys. 303, 165 (2004).

${ }^{27}$ E. A. Rohlfing and J. E. M. Goldsmith, J. Opt. Soc. Am. B 7, 1915 (1990).

${ }^{28}$ M. Izuha and K. Yamanouchi, Chem. Phys. Lett. 242, 435 (1995).

${ }^{29}$ A. Van Orden and R. J. Saykally, Chem. Rev. 98, 2313 (1998).

${ }^{30}$ G. Monninger, M. Förderer, P. Gürtler, S. Kalhofer, S. Petersen, L. Nemes, P. G. Szalay, and W. Krätschmer, J. Phys. Chem. A 106, 5779 (2002).

${ }^{31}$ S. Saha and C. M. Western, J. Chem. Phys. 125, 224307 (2006).

${ }^{32}$ F. J. Northrup and T. J. Sears, J. Opt. Soc. Am. B 7, 1924 (1990). 
${ }^{33}$ R. A. Whiteside, R. Krishnan, M. J. Frisch, J. A. Pople, and P. Von R. Schleyer, Chem. Phys. Lett. 80, 547 (1981).

${ }^{34}$ H. Fueno and Y. Taniguchi, Chem. Phys. Lett. 312, 65 (1999).

${ }^{35}$ A. Terentyev, R. Scholz, M. Schreiber, and G. Seifert, J. Chem. Phys. 121, 5767 (2004)

${ }^{36}$ H. Sasada, T. Amano, C. Jarman, and P. F. Bernath, J. Chem. Phys. 94, 2401 (1991).

${ }^{37}$ D. W. Tokaryk and S. Civis, J. Chem. Phys. 103, 3928 (1995).

${ }^{38}$ M. Tulej, M. Meisinger, G. Knopp, A. M. Walser, T. Gerber, and P. P. Radi, J. Raman Spectrosc. 38, 1022-1031 (2007).

${ }^{39}$ M. Tulej, G. Knopp, T. Gerber, and P. P. Radi, J. Raman Spectrosc. 41, 853-858 (2010).

${ }^{40}$ H. Linnartz, Phys. Scr. 69, C37 (2004).

${ }^{41}$ T. Müller and P. H. Vaccaro, Chem. Phys. Lett. 266, 575 (1997).

${ }^{42}$ P. Radi, H.-M. Frey, B. Mischler, A. Tzannis, P. Beaud, and T. Gerber, Chem. Phys. Lett. 265, 271 (1997).

${ }^{43}$ S. Williams, J. D. Tobiason, J. R. Dunlop, and E. A. Rohlfing, J. Chem. Phys. 102, 8342 (1995).

${ }^{44}$ S. Williams, E. A. Rohlfing, L. A. Rahn, and R. N. Zare, J. Chem. Phys. 106, 3090 (1997).
${ }^{45}$ Y. Prior, IEEE J. Quantum Electron. 20, 37 (1984).

${ }^{46}$ T. K. Yee and T. K. Gustafson, Phys. Rev. A 18, 1597 (1978).

${ }^{47}$ T. A. W. Wasserman, P. H. Vaccaro, and B. R. Johnson, J. Chem. Phys. 108, 7713 (1998)

${ }^{48}$ D. Cooper and J. Jones, J. Quant. Spectrosc. Radiat. Transf. 22, 201 (1979).

${ }^{49}$ S. Williams, R. N. Zare, and L. A. Rahn, J. Chem. Phys. 101, 1093 (1994).

${ }^{50}$ B. McCall, R. Casaes, M. Ádámkovics, and R. Saykally, Chem. Phys. Lett. 374, 583 (2003).

${ }^{51}$ H.-J. Werner, P. J. Knowles, G. Knizia, F. R. Manby, M. Schütz et al., MOLPRO, version 2011.1, a package of ab initio programs, 2011, see http://www.molpro.net.

${ }^{52}$ H.-J. Werner and W. Meyer, J. Chem. Phys. 74, 5794 (1981).

${ }^{53}$ H.-J. Werner and P. J. Knowles, J. Chem. Phys. 82, 5053 (1985).

${ }^{54}$ P. J. Knowles and H.-J. Werner, Chem. Phys. Lett. 145, 514-522 (1988).

${ }^{55}$ H.-J. Werner and P. J. Knowles, J. Chem. Phys. 89, 5803-5814 (1988).

${ }^{56}$ H.-J. Werner and P. J. Knowles, Theor. Chim. Acta 78, 175 (1990).

${ }^{57}$ W. Weltner and D. McLeod, J. Chem. Phys. 40, 1305 (1964).

${ }^{58} \mathrm{C}$. M. Western, PGOPHER, a program for simulating rotational structure 2012.

${ }^{59}$ A. Mebel and R. Kaiser, Chem. Phys. Lett. 360, 139 (2002). 